\title{
Monitoramento da temperatura atmosférica visando o conforto térmico e a conservação de florestas
}

\author{
Monitoring air temperature for the thermal comfort and conservation of forests
}

\author{
Álvaro Boson de Castro-Faria \\ Universidade Tecnológica Federal do Paraná, Dois Vizinhos, PR, Brasil. Autor para correspondência: alvarob@utfpr.edu.br
}

Submissão: 11/10/2020 / Aceite: 14/12/2020

\begin{abstract}
RESUMO
As ondas de calor têm despertado a preocupação quanto aos danos que ocasionam sobre os serviços ecossistêmicos, e combinadas com estiagens, o excesso de calor tem favorecido o aumento da mortalidade das árvores mundo afora. Partindo-se da hipótese em que a região sudoeste do Paraná, apresenta condições favoráveis à ocorrência de anomalias climáticas caracterizadas como ondas de calor de verão, este trabalho teve como objetivo geral, observar o comportamento da temperatura atmosférica no município de Dois Vizinhos, para o interstício entre 2018 a 2020, e em específico: i) analisar quais os intervalos dos horários de verão em Dois Vizinhos, a temperatura atmosférica se apresenta mais elevada; ii) verificar por meio do índice proposto de estresse florestal (forest-based stress index ou FBS), os eventos caracterizados como de onda de calor, entre os anos de 2018 a 2020; iii) discutir a viabilidade do índice FBS, tendo como critério, outros índices de monitoramento ambiental; iv) apresentar argumentos sobre aspectos ecofisiológicos e de conservação florestal. As conclusões foram que o período noturno entre $21 \mathrm{~h}-03 \mathrm{~h}$ de 2020 , da estação de verão de Dois Vizinhos, foi significativamente mais quente, no triênio analisado. O horário entre $15 \mathrm{~h}-21 \mathrm{~h}$ de 2020 foi significativamente mais quente, entre as categorias horarias analisadas. Por meio do índice FBS de estresse florestal, verificou-se o total de 36 episódios caracterizados como de Ondas de Calor na localidade, inclusive noturnas, entre os anos de 2018 a 2020.
\end{abstract}

PALAVRAS-CHAVE: floresta atlântica, el niño, estiagens, proteção da saúde florestal, índice de estresse florestal (FBS), ondas de calor.

\begin{abstract}
Heat waves have raised concerns about the damage they do to ecosystem services, and combined with droughts, excessive heat has favored the rise in tree mortality worldwide. Based on the hypothesis that the southwestern region of the state of Paraná presents suitable conditions for the occurrence of anomalous weather events characterized as summer heat waves, this study was conducted to observe air temperature patterns in the municipality of Dois Vizinhos between 2018 and 2020. Its specific objectives were to i) determine which periods of the day during the summer in Dois Vizinhos registered the highest air temperatures; ii) verify the events characterized as heat waves between the years 2018 and 2020 using the proposed forest-based stress index (FBS); iii) discuss the feasibility of the FBS index by making reference to other environmental monitoring indices; and iv) present arguments on aspects of ecophysiology and forest conservation. The results showed that the night period between 9 p.m. and 3 a.m. in Dois Vizinhos in summer 2020 was significantly hotter in the three years of the study. The interval between 3 p.m. and 9 p.m. in summer 2020 was significantly hotter among the hourly categories recorded. According to the FBS index, there were a total of 36 episodes characterized as Heat Waves in this region, including the nighttime.
\end{abstract}

KEYWORDS: Atlantic Forest, El Niño, droughts, forest health protection, forest-based stress index (FBS), heat waves.

\section{INTRODUÇÃO}

O termo onda de calor $(\mathrm{OC})$ é utilizado para descrever um período prolongado de clima quente extremo, incomum e desconfortável (ALVES et al. 2016). Para a Organização Meteorológica Mundial (WMO 2015), este complexo fenômeno resulta da interação entre a radiação solar, da temperatura e umidade atmosféricas; e, da ação dos ventos. Para FIRPO et al. (2012), três parâmetros devem ser considerados ao 
se analisar as OC: a persistência; a temperatura atmosférica; e, a frequência. As OC ocorrem ao redor do mundo e tem sido mais reportada desde o ano de 2000, por estar causando danos à saúde humana e tragédias ambientais (BITENCOURT et al. 2016, KOGIMA \& ELY 2019, BURAS et al. 2020).

Em termos globais, KIRKPATRICK \& LEWIS (2020) constataram o aumento da ocorrência de OC, desde a década de 1950. Enquanto a maioria dos estudos no tema tenham dado ênfase aos efeitos durante o dia, existe um interesse emergente em conhecer, também, os efeitos durante os períodos noturnos (WMO 2015). É previsto o aumento de danos por OC no decorrer do século XXI, sobretudo na estação do verão (ALVES et al. 2016). Ondas de calor em combinação com estiagens, estão intrinsecamente ligadas, produzindo efeitos intensificados, pois, a diminuição das chuvas está associada com à redução da cobertura de nuvens, que aumenta o fluxo de radiação para o solo, reduzindo a umidade do ar, e aumentando ainda mais a sensação de desconforto térmico (TESKEY et al. 2015).

As ondas de calor também têm despertado a preocupação quanto aos danos que ocasionam sobre os serviços ecossistêmicos (CARDONA et al. 2012). Combinadas com estiagens, o excesso de calor tem favorecido o aumento da mortalidade das árvores mundo afora. Em muitos casos, após estarem estressadas, elas começam a ser atacadas por agentes bióticos, e, considerando a planta como um todo, o excesso de calor pode induzir a redução do desenvolvimento da área foliar e do crescimento (ALLEN et al. 2010). Processos, como a respiração e a transpiração, são afetados, mas, principalmente, a fotossíntese é prejudicada.

Os efeitos da temperatura atmosférica sobre o bioma da floresta atlântica, especialmente protegido, deve ser mais bem compreendido. A sudoeste do Paraná, na localidade de Dois Vizinhos, apresenta-se com fitofisionomia de ecótono entre Floresta Estacional Semidecidual (FES) e Floresta Ombrófila Mista (FOM), onde o clima preponderante se apresenta com elevada amplitude, e, com inversões térmicas. Acredita-se, pois, que nesta mesorregião, as espécies florestais estejam vulneráveis ao estresse e ao declínio por OC, sobretudo nos horários mais quentes do verão, inclusive noturnos, e, agravados por condições de secas. Há a necessidade de estudar estes padrões, no sentido de avaliar os danos ambientais por causas naturais, e buscar medidas de mitigação ambientais e conservacionistas, a serem tomadas em casos de extremos climáticos.

Partindo-se da hipótese em que a região sudoeste do Paraná, apresenta condições favoráveis à ocorrência de anomalias climáticas caracterizadas como ondas de calor de verão, este trabalho teve como objetivo geral, observar o comportamento da temperatura atmosférica no município de Dois Vizinhos, para o interstício entre 2018 a 2020, e em específico: i) analisar quais os intervalos dos horários de verão em Dois Vizinhos, a temperatura atmosférica se apresenta mais elevada; ii) verificar por meio do índice proposto FBS, os eventos caracterizados como de onda de calor, entre os anos de 2018 a 2020; iii) discutir a viabilidade do índice FBS, tendo como critério, outros índices de monitoramento ambiental; iv) apresentar argumentos sobre aspectos ecofisiológicos e de conservação florestal.

\section{MATERIAL E MÉTODOS}

A colonização da região sudoeste do Paraná ocorreu por meio de processos de supressão descontrolada de vegetação nativa. TOMASETTO et al. (2009) citam que a região possui 42 municípios, e do total das propriedades rurais, $87 \%$ são familiares e destas, $94 \%$ possuem áreas inferiores a 50 ha. Em sua maioria, estas propriedades rurais produzem alimentos para o abastecimento dos próprios municípios e nas quais, também, se destaca a pecuária leiteira.

O clima da região, segundo a classificação de Köppen, é o Cfa (úmido, com verões quentes), com temperatura atmosférica média no mês mais frio, entre -3 a $18 \stackrel{\circ}{\circ} \mathrm{C}$, e, com precipitação pluviométrica mensal superior a 40 milímetros (ALVARES et al. 2013).

O município analisado de Dois Vizinhos, especificamente, possui uma área de cerca de 41,8 mil hectares, com altitude em torno de 360 metros. Existem atualmente, apenas 2,2\% desta área, que se apresenta, ainda, com remanescentes do bioma da mata atlântica, e, que equivalem ao total de 923,1 hectares, sendo que destas, 527,2 ha são de Floresta Estacional Semidecidual, e, 395,87 hectares, são de Floresta Ombrofila Mista (FOM), o que caracteriza a localidade como sendo um ecótono entre FES e FOM (Figura 1). É comum verificar a presença do pinheiro do Paraná (Araucaria angustifolia Bentol Kuntze, listada como ameaçada de extinção, com sua ocorrência integrada nestas fitofisionomias, e, ocorrendo de forma isolada. E, de forma geral, todo o bioma de floresta atlântica remanescente, permanece bastante vulnerável à processos de antropização, a depender da conscientização ambiental dos pequenos produtores rurais.

Optou-se por trabalhar com os dados climáticos de verão, representados pelos meses de janeiro, 
fevereiro e março, e, com o interstício compreendido entre os anos de 2018 a 2020, e, em relação à média para cada três dias $(72 \mathrm{~h}$ ). Foram duas hipóteses testadas, a primeira, de que o horário compreendido entre 15 a $21 \mathrm{~h}$, é o mais suscetível ao calor, e, a segunda, de que estaria ocorrendo o estresse por OC durante o período noturno, compreendido entre $21 \mathrm{~h}$ às $3 \mathrm{~h}$, com ênfase para o verão de 2020 . Os dados foram agrupados em composições, totalizando quatro possibilidades horárias, ou seja, das 15 às $21 \mathrm{~h}$, das 21 às $03 \mathrm{~h}$, das 03 às $09 \mathrm{~h}$, e, das 09 às $15 \mathrm{~h}$. Este agrupamento permitiu a obtenção de séries anuais com 30 repetições. Foram empregados os parâmetros climáticos da estação automática instalada em Dois Vizinhos, cedidos pelo Instituto Nacional de Meteorologia (INMET), localizada dentro do Câmpus da UTFPR. Os parâmetros utilizados foram: temperatura do ar instantânea (TAl), e, a temperatura do ponto de orvalho (TPO).

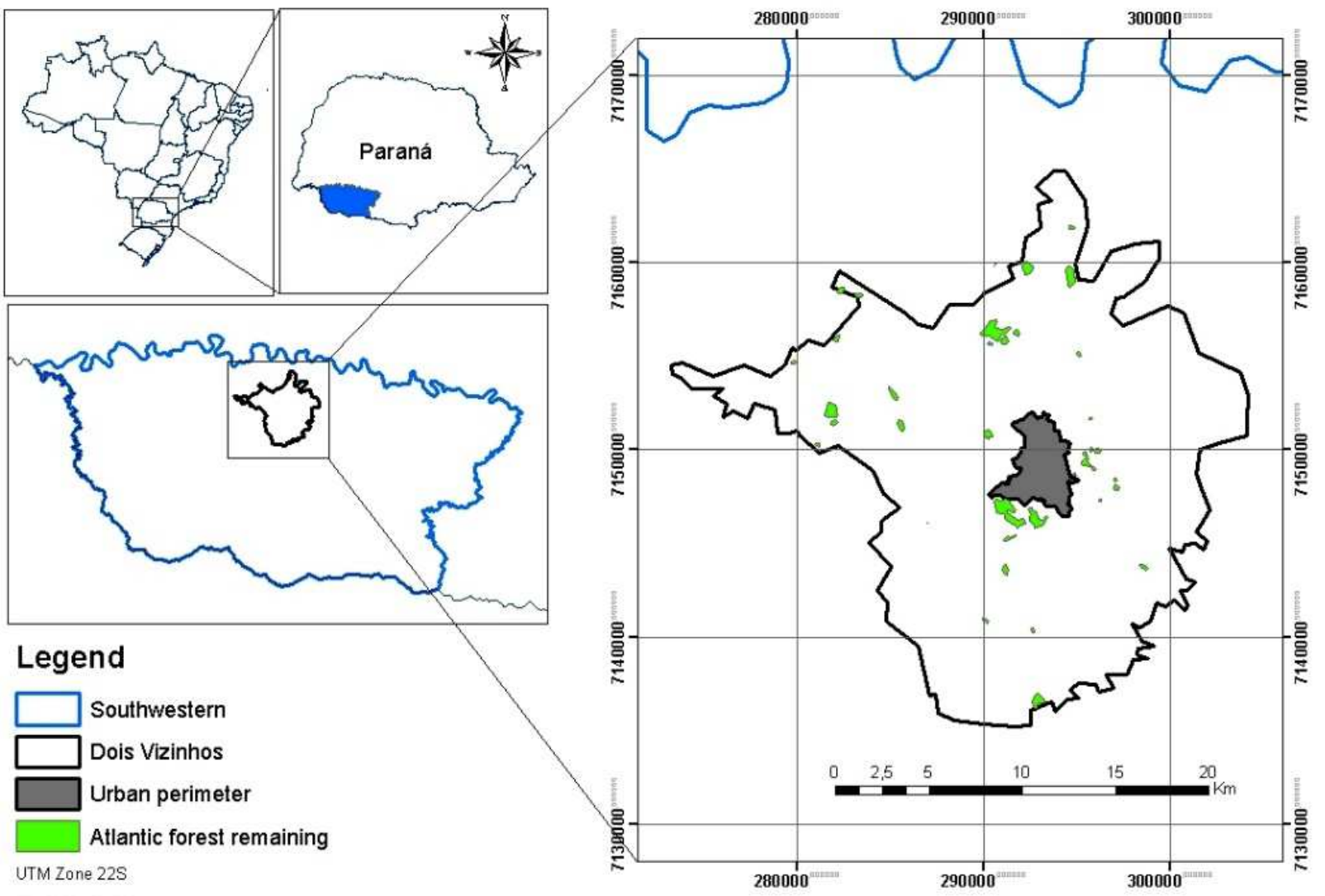

SIRGAS 2000 Datum

Figura 1. Identificação do município de estudo e dos fragmentos de floresta atlântica, especialmente protegidos por lei.

Figure 1. The municipality identified in the study and the remaining Atlantic Forest, which has special legal protection.

Os dados utilizados eram homogêneos, e foram utilizados na estimação dos intervalos de confiança mínimos e máximos a 95\% de probabilidade. Neste trabalho, se propõe o índice de estresse florestal, ou, Forest-Based Stress Index (FBS), adimensional, utilizando como variáveis de entrada, a temperatura atmosférica instantânea (TAI), e sua respectiva temperatura de ponto de orvalho (TPO), que é aquela na qual o ar, resfriado sob pressão constante, torna-se saturado. Este parâmetro indicaria, na prática, o efeito do vapor de água, sobre a temperatura da atmosfera, e assim, indiretamente, o efeito ambiental natural sobre a transpiração das plantas. A lógica é a de que, se a TPO estiver equivalente a própria TAI, e, sendo o resultado da divisão entre estes dois parâmetros, o valor igual a 1,0, haveria a indicação de uma situação de saturação do ar (umidade relativa igual a 100\%), e, indicando a ausência de estresse vegetal devido à transpiração. Isto é, as plantas não estariam perdendo água para o meio atmosférico, e, assim, caracterizando uma situação de conforto térmico, com a potencialização da absorção da energia solar pela fotossíntese. Ainda, nos casos de ausência da luz (monitorados à noite), o resultado indicaria a potencialização do processo de respiração, e, consequentemente, de crescimento vegetal (Equação 1). Esta mesma situação, também, pode ser representada por meio da Equação 2, que utiliza uma 
transformação, com resultados variando de 1 a 10. Neste ajuste, a partir da escala logarítmica de base natural neperiana, quanto maior a temperatura atmosférica, maior o FBS, e, a nota máxima de valor 10, indicaria o pior cenário para e estresse vegetal.

$$
\begin{aligned}
& F B S=\frac{T P O}{T A I} \\
& F B S=10+\ln \left(1-\frac{T P O}{T A I}\right)
\end{aligned}
$$

Em que: FBS: 1) índice de estresse vegetal por ondas de calor (adimensional); TPO: temperatura do ponto de orvalho instantânea $\left({ }^{\circ} \mathrm{C}\right)$; TAl: temperatura atmosférica instantânea $\left({ }^{\circ} \mathrm{C}\right)$. 2) Modelo para o FBS logarítmico, e que resulta em valor mínimo de 1, e, máximo equivalente a 10.

A inferência sobre a existência de OC foi constatada a partir do FBS estimado para o intervalo horário, e, por meio do critério da média superior a duas vezes o erro padrão, e, para OC extremas, o da média superior a três vezes o erro padrão.

\section{RESULTADOS}

Para o triênio analisado, as séries indicaram que as temperaturas médias foram mais amenas para o horário das 3 às $9 \mathrm{~h}$, como era esperado, devido às madrugadas $\left(20,30^{\circ} \mathrm{C} ; 20,48{ }^{\circ} \mathrm{C}\right.$; e, $\left.20,68{ }^{\circ} \mathrm{C}\right)$, já, para os horários entre 9-15h e 21-03h, em 2018 e 2019, não houve diferenças significativas. E, em 2020, se comprovou que as noites foram significativamente mais quentes, se comparado aos horários entre 3 às $15 \mathrm{~h}$ $\left(24,49^{\circ} \mathrm{C} ; \mathrm{F}=127,05, \mathrm{p}<0,05\right)$. A Tabela 1 indica que em todos os três anos observados, o horário das $15-$ $21 \mathrm{~h}$ foi o que sempre se apresentou mais quente $\left(26,95^{\circ} \mathrm{C} ; 27,90{ }^{\circ} \mathrm{C}, 28,60{ }^{\circ} \mathrm{C}\right)$.

Tabela 1. Diferenças observadas por meio da Anova, e, médias testadas.

\begin{tabular}{|c|c|c|c|c|}
\hline ANO & HORA & TAI & TPO & FBS (In) \\
\hline \multirow[t]{6}{*}{2018} & 03:00-08:59 & $20,30 a$ & $18,68 \mathrm{a}$ & $7,12 \mathrm{a}$ \\
\hline & 09:00-14:59 & $22,13 \mathrm{~b}$ & $19,06 \mathrm{a}$ & $7,84 \mathrm{~b}$ \\
\hline & 21:00-02:59 & $23,17 b$ & $19,14 a$ & $7,94 \mathrm{~b}$ \\
\hline & 15:00-20:59 & $26,95 \mathrm{c}$ & $19,39 \mathrm{a}$ & $8,59 \mathrm{c}$ \\
\hline & $\mathrm{F}$ (trat.) & $90,83^{\star *}$ & $0,73 \mathrm{~ns}$ & $15,74^{\star \star}$ \\
\hline & CV (\%) & 6,97 & 9,88 & 10,54 \\
\hline \multirow[t]{6}{*}{2019} & 03:00-08:59 & $20,48 a$ & $18,57 \mathrm{a}$ & $7,53 \mathrm{a}$ \\
\hline & 09:00-14:59 & $22,80 \mathrm{~b}$ & $19,12 \mathrm{a}$ & $8,06 \mathrm{~b}$ \\
\hline & $21: 00-02: 59$ & $23,53 \mathrm{~b}$ & $19,21 \mathrm{a}$ & $8,11 \mathrm{~b}$ \\
\hline & 15:00-20:59 & $27,90 \mathrm{c}$ & $19,47 \mathrm{a}$ & $8,71 \mathrm{c}$ \\
\hline & $\mathrm{F}$ (trat.) & $74,55^{\star *}$ & $1,02 \mathrm{~ns}$ & $23,43^{\star \star}$ \\
\hline & CV (\%) & 8,30 & 10,77 & 6,77 \\
\hline \multirow[t]{6}{*}{2020} & 03:00-08:59 & $20,68 \mathrm{a}$ & $16,70 \mathrm{a}$ & $8,26 \mathrm{a}$ \\
\hline & 09:00-14:59 & $22,40 \mathrm{~b}$ & $17,36 \mathrm{a}$ & $8,40 a b$ \\
\hline & 21:00-02:59 & $24,49 \mathrm{c}$ & $17,41 \mathrm{a}$ & $8,66 \mathrm{~b}$ \\
\hline & 15:00-20:59 & $28,60 d$ & $17,60 \mathrm{a}$ & $9,00 \mathrm{c}$ \\
\hline & $\mathrm{F}$ (trat.) & $127,05^{\star \star}$ & $0,641 \mathrm{~ns}$ & $17,53^{\star \star}$ \\
\hline & CV (\%) & 6,89 & 15,58 & 4,97 \\
\hline
\end{tabular}

Table 1. Differences observed through ANOVA and the means tested.

Em que: $\mathrm{F}$ = Valor F calculado; CV (\%): coeficiente de variação; ${ }^{* *}=$ significativo ao nível de $1 \%$ de probabilidade $(p<0,01)$; ns: não significativo $(p>=0,05)$. 
Entre o período observado, e, para todos os horários, não houve diferenças significativas no parâmetro da temperatura de ponto de orvalho. Os valores variaram entre $18,57{ }^{\circ} \mathrm{C}$ a $19,47{ }^{\circ} \mathrm{C}$ em $2018 \mathrm{e}$ 2019. Já em 2020 , ano mais quente, a TPO ficou entre $16,70^{\circ} \mathrm{C}$ a $17,60^{\circ} \mathrm{C}$, em função, provavelmente, do efeito atenuante do calor sobre a pressão atmosférica, diminuindo-a.

A análise, integrando-se a TAI e a TPO, indicou o efeito da umidade na sensação térmica, e, no estresse vegetal. Isto é, por meio do índice FBS, se verificou que o horário mais ameno se deu entre 3-9h, em todos os três anos verificados. Algo que era esperado. Porém, também se esperava verificar uma diferença significativa de estresse em relação aos horários das 9-15h, e das 21-3h. Para o triênio, não houve diferenças significativas. Mas, o FBS no horário entre 15-21h restou significativamente mais elevado, nas três sequências observadas. Por exemplo, o FBS de 2020 neste horário, alcançou a cota numérica de nove pontos, significativamente superior aos outros horários $(F=17,53, p<0,05)$.

Tendo-se constatado que o horário entre 15-21h demonstrou o maior estresse vegetal, se apresenta, nas Tabelas 2 e 3, os parâmetros descritivos gerais do FBS, para este período, e, para esta classe horária, para a definição dos limites de OC, na localidade.

Tabela 2. Dados descritivos do FBS entre 15-21h e nos anos entre 2018 a 2020.

Table 2. FBS data for the period between 3 p.m. and 9 p.m. during 2018-2020.

\begin{tabular}{lc}
\hline & Índice FBS (15h-21h) \\
\hline Mínimo & 6,78 \\
\hline Máximo & 9,52 \\
\hline Amplitude & 2,74 \\
\hline Média & 8,77 \\
\hline Variância & 0,20 \\
\hline Desvio & 0,45 \\
\hline CV & 0,05 \\
\hline GL & 89 \\
\hline t tabelado $p<0,05$ & 2,807 \\
\hline Erro padrão da média $(\mathrm{n}=90)$ & 0,0479 \\
\hline $\mathrm{t}^{\star}$ erro padrão & 0,1343 \\
\hline IC máx (p<0,05) & 8,90 \\
\hline IC mín (p<0,05) & 8,63 \\
\hline Onda de Calor & 9,04 \\
\hline Onda de Calor extrema & 9,17 \\
\hline
\end{tabular}

Em que: IC máx = Valor máximo para o Intervalo de Confiança da média, ao nível de 95\% de probabilidade; IC mín = Valor mínimo para o Intervalo de Confiança da média, ao nível de 95\% de probabilidade.

Tabela 3. Índice FBS ajustado para o município de Dois Vizinhos, PR.

Table 3. FBS index adjusted for the municipality of Dois Vizinhos, PR.

\begin{tabular}{cc}
\hline \multicolumn{1}{c}{ FBS } & Interpretação \\
\hline$<9,04$ & $\begin{array}{c}\text { Ondas de Calor inexistentes; continuar o monitoramento da média } \\
\text { móvel diariamente. }\end{array}$ \\
\hline Entre 9,04 a 9,17 & Existência de ondas de calor; medidas de mitigação ambientais são \\
& necessárias; seguir com o monitoramento. \\
\hline$>9,17$ & Ondas de calor extremas; medidas intermitentes de mitigação \\
ambientais são necessárias; seguir com o monitoramento.
\end{tabular}


Em que pese a média geral do triênio tenha sido de 8,77, verifica-se que as ondas de calor em Dois Vizinhos, podem ser caracterizadas quando o FBS, resultar o valor mínimo de 9,04, e, que ondas de calor extremo podem ser verificadas com o resultado do índice, a partir da cota de 9,17. Com toda a distribuição horária do triênio, a Figura 2, indica a ocorrência de OC, com a linha de base a partir de 9,04, para os verões de 2018 a 2019.

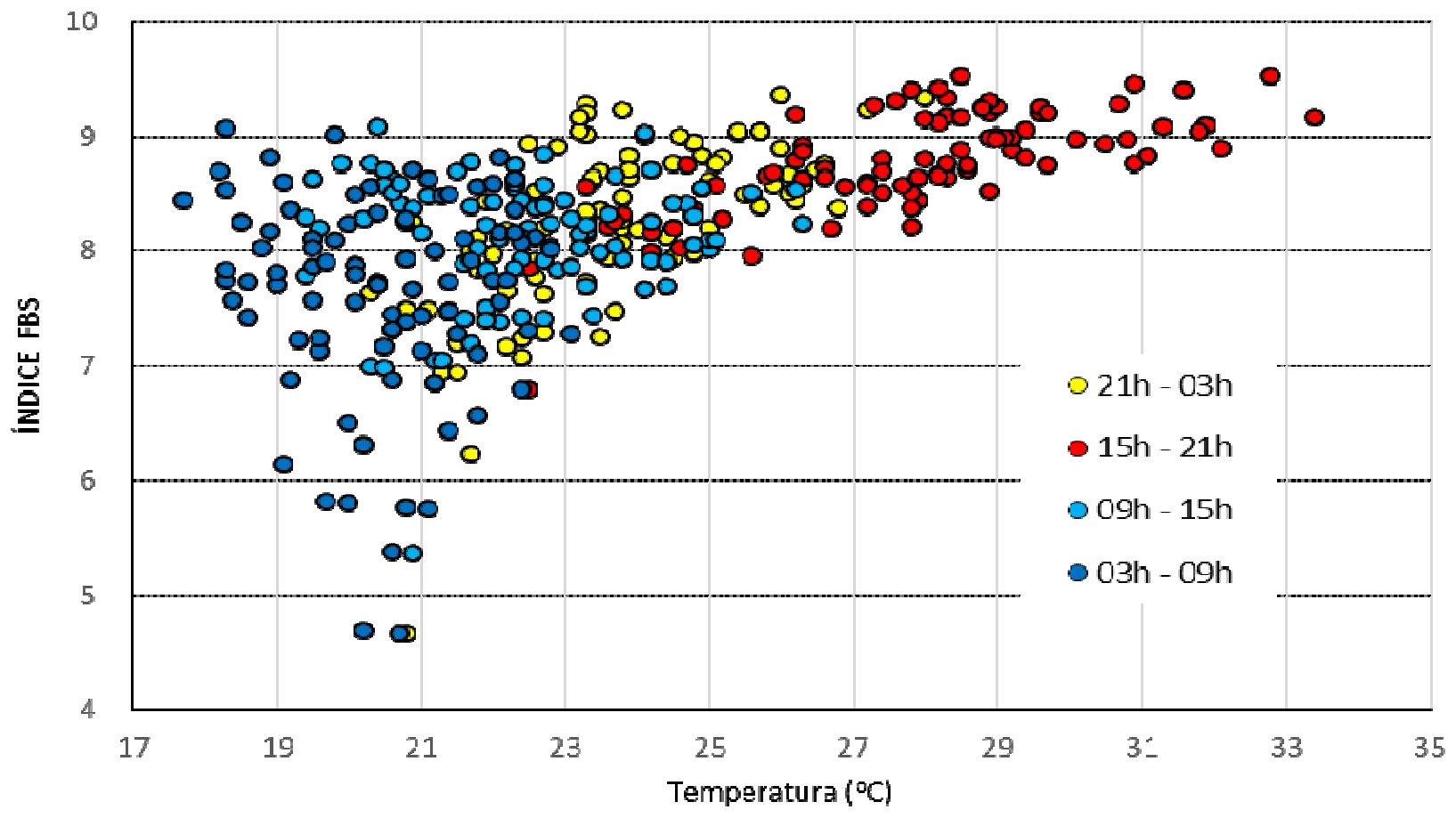

Figura 2. Comportamento geral do Índice FBS, entre 2018 a 2020.

Figure 2. Forest-based stress index (FBS) for 2018-2020.

Foram 36 episódios, caracterizados como de calor e de calor extremo, para o horário entre 15-21h. A distribuição, também organizada por meio da Tabela 4, reitera que o calor no ano de 2020, de fato, foi mais impactante. Do total de ocorrências para o triênio, $75 \%(52,8 \%+22,2 \%)$ ocorreram no horário das 15 às $21 \mathrm{~h}$. Também se observa episódios de estresse por calor no horário noturno entre as 21 -3h, com sete episódios. Para os outros horários, a ocorrência pode ser considerada esporádica.

Tabela 4. Distribuição dos episódios de calor, registrados na série.

Table 4. Distribution of the heat episodes recorded in the series.

\begin{tabular}{|c|c|c|c|c|c|c|}
\hline & Horário & 2018 & 2019 & 2020 & Soma & $\%$ \\
\hline \multirow{4}{*}{ Onda de Calor } & $03 \mathrm{~h}$ a $09 \mathrm{~h}$ & - & - & 1 & 1 & $2,8 \%$ \\
\hline & $09 \mathrm{~h}$ a $15 \mathrm{~h}$ & - & - & 1 & 1 & $2,8 \%$ \\
\hline & $21 \mathrm{~h}$ a $03 \mathrm{~h}$ & - & - & 1 & 1 & $2,8 \%$ \\
\hline & $15 \mathrm{~h}$ a $21 \mathrm{~h}$ & - & 6 & 2 & 8 & $22,2 \%$ \\
\hline \multirow{6}{*}{ Onda de Calor Extremo } & 03h a 09h & - & - & - & - & $0,0 \%$ \\
\hline & $09 \mathrm{~h}$ a $15 \mathrm{~h}$ & - & - & - & - & $0,0 \%$ \\
\hline & $21 \mathrm{~h}$ a $03 \mathrm{~h}$ & - & 1 & 5 & 6 & $16,7 \%$ \\
\hline & $15 \mathrm{~h}$ a $21 \mathrm{~h}$ & 6 & 1 & 12 & 19 & $52,8 \%$ \\
\hline & Soma & 6 & 8 & 22 & 36 & \\
\hline & $\%$ & $16,7 \%$ & $22,2 \%$ & $61,1 \%$ & & \\
\hline
\end{tabular}

\section{DISCUSSÃO}

O índice FBS proposto vai de encontro com os pressupostos por GEIRINHAS (2016), que menciona que, as características para um bom índice que se pretenda monitorar o calor, deve apresentar primeiramente, dados facilmente disponíveis; e, devem ser relevantes para os ecossistemas e a atividade 
econômica; devem representar vastas regiões, de uma forma consistente e permitindo a sua comparação; também, devem ter sensibilidade às variações naturais e antropogênicas do clima.

Para RADINOVIÉ \& CURIÉ (2012), a caracterização de episódios de calor prolongado, é realizada a partir de dados de temperaturas máximas, em agrupamentos de três e cinco dias, por meio da verificação das médias somadas a duas vezes o desvio padrão. Já o FBS ora proposto, foi calculado com a temperatura instantânea e a de ponto de orvalho. Entende-se que este parâmetro representa melhor a evolução diária e horária do calor em uma determinada localidade, e a temperatura máxima, apresentaria os picos diários, mas, com menor variabilidade, isto é, para o monitoramento em periodicidades dilatadas, as médias instantâneas seriam mais apropriadas.

Vale frisar, que caso tivesse sido utilizado o desvio padrão e não o erro padrão na estimação dos limites de calor, a cauda estaria apresentando o limite caracterizado como de OC, a partir da cota de 9,67 $\left(=8,77+2^{*} 0,45\right)$ na caracterização dos episódios, mesmo que o máximo observado tenha sido o valor de 9,52. Neste caso, para a área de estudo, não teria sido caracterizado, nenhum episódio de onda de calor. Ainda, caso o valor de caracterização fosse com três vezes o desvio, o limite resultaria em valor superior ao máximo da cota numérica de dez, que, é o valor máximo considerando a lógica do FBS. Apesar de ambos tratarem sobre a variação da média, o desvio trata da dispersão da amostra em relação a média, enquanto o erro padrão ajuda se Ihe avaliar a confiabilidade.

A recomendação da Organização Mundial Meteorológica, emprega a temperatura máxima, somada de 5 graus, em relação as médias para a primavera e para o verão. O estabelecimento de valores de calor por meio de índices, cujos limites mínimos, represente o percentil mínimo de 95\% da normal, também vai de encontro com as recomendações desta organização (WMO 2015). Neste sentido, o FBS acaba por ser mais conservador, pois ao utilizar a temperatura média instantânea associada ao erro padrão, acaba por caracterizar as ondas de calor em temperaturas inferiores. Mas, com episódios que ocorrem com maior frequência, no decorrer das estações, em comparação com os pressupostos da WMO.

De acordo com o Painel Intergovernamental de Mudanças Climáticas (IPCC 2014), em muitas áreas do globo, os dias e noites quentes intensos têm sido mais frequentes, e o período entre 1995 a 2006, apresentou a maior temperatura média global desde 1850. Para o Brasil, MARENGO (2014) informa que as chuvas tendem a continuar diminuindo durante o século XXI, sendo as reduções mais intensas no Nordeste e na Amazônia, sendo que, as projeções indicam aumento da temperatura e de extremos de calor, bem como redução na frequência de geadas devido ao aumento da temperatura mínima, principalmente nos estados do Sudeste, Sul e Centro-Oeste. Para VINCENT et al. (2005), existe a tendência no aumento da frequência de ocorrência das noites mais quentes, principalmente no verão. Anomalias climáticas no Paraná foram reportadas por SILVA et al. (2015), por meio de indicador que apresentava o número máximo de dias secos consecutivos no ano. Estes autores demonstraram que em Pato Branco e em Planalto (na região sudoeste do Estado), as estiagens estão mais frequentes. KOGIMA \& ELY (2019), indicaram que, no Paraná, dentre as cidades mais quentes deste estado, estão Planalto, Palotina, Fernandes Pinheiro, Cambará, Bandeirantes, Bela Vista do Paraiso e Londrina.

Todas as menções indicam que a temperatura do sudoeste paranaense, uma região quente, e que apresenta elevada amplitude térmica, merece ser monitorada visando o estresse que o calor poderia causar tanto para a sociedade, quanto nos setores agrícolas, e, na conservação dos remanescentes florestais nativos.

CASTRO FARIA et al. (2019), previram que o sudoeste paranaense apresentaria uma estiagem severa até o inverno do ano de 2021, o que foi confirmado já no verão de 2020. Em Dois Vizinhos, mesma mesorregião, VIEIRA et al. (2018) demonstraram que as chuvas na estação quente, são as mais escassas no decorrer do ano. Recomendam-se novas pesquisas que estejam indicando para a sociedade, os episódios de calor, e a necessidade de adoção de medidas de mitigação ambiental, a exemplo do uso dos sistemas de irrigação. O problema torna-se mais significativo ao se pensar a mitigação do calor para as florestas nativas. O que deveria ser observado pelas autoridades, seria a urgência em promover a restauração das áreas de preservação permanente, no âmbito da lei federal de proteção da vegetação nativa, ou ainda, a implementação massiva dos Planos Municipais de Mata Atlântica, previstos pela lei que Ihe conferiu regime especial de proteção. Assim, o aumento de áreas restauradas poderia com o tempo, favorecer a formação de nuvens por meio da transpiração das plantas, dentre outros serviços ambientais que as florestas proporcionam, e, em larga escala, contribuindo com que o clima local se torne menos estressante.

As massas de ar que influenciam o clima na região Sul estão condicionadas aos efeitos dos sistemas de formação anormais entre oceano e atmosfera conhecido com El Niño de oscilação sul (ENOS), 
decorrente do aquecimento do oceano Pacífico, e o seu inverso La Niña. Anos com El Niño resultam em OC são mais intensas no inverno, e, nos meses mais quentes, se apresentam mais intensas quando ocorre a $L a$ Niña (FIRPO et al. 2012). Para MORALES et al. (2020), secas mais severas, e enchentes, foram comprovadas para a América do Sul, a partir da metade do século XX, estando fortemente relacionadas aos eventos de ENOS.

ALVES et al. (2016) adotaram o índice denominado temperatura-umidade (ITU), para o monitoramento do calor, integrando parâmetros com unidades distintas. Porém, entendeu-se que este parâmetro não possui uma unidade interessante para o monitoramento do calor, que pudesse representar 0 efeito sobre as plantas.

Existem três processos que condicionam o desenvolvimento das plantas, a transpiração, a fotossíntese e a respiração. Procurar manejá-los, visando o bem-estar das árvores contra a ocorrência das ondas de calor, pode contribuir tanto para a garantia da produção de serviços ecossistêmicos, quanto para se lhes favorecer o crescimento, e por que não dizer, a produtividade.

Em linhas gerais, sabe-se que as árvores dissipam calor pela transpiração, para equilibrarem a temperatura interna com o meio atmosférico. O vapor d'água, ao evaporar pelos estômatos, diminui a temperatura na superfície foliar e o estresse vegetal. Quanto maior a temperatura atmosférica, maior será a transpiração, e haverá maior desconforto térmico quando não houver mais água no solo disponível para este processo. Tem-se como regra que o estresse maior será com uma temperatura acima de $35 \stackrel{\circ}{\circ}$, onde as árvores fecharão seus estômatos, e entrarão naturalmente em dormência fisiológica, e, com isso, deixando de realizar a fotossíntese. À noite, se houver estresse térmico, as árvores deixarão de respirar, ou, se estiverem demandando carboidratos não produzidos durante o dia, a tendência será de aumento de susceptibilidade de ataques de pragas e doenças, podendo entrar em declínio (ALLEN 2009).

Estudos sobre a vulnerabilidade de ecossistemas tem ganhado relevância científica, com o objetivo de monitorar padrões e tendências da mortalidade de árvores induzida pelo clima (HARTMANN et al. 2015, AUBIN et al. 2018, HARTMANN et al. 2018). A detecção do nível de intensificação dos estresses nas florestas nativas torna-se um desafio cada vez mais importante para a sociedade, uma vez que estas podem levar décadas para restaurarem os serviços ecossistêmicos que providenciaram (TRUMBORE et al. 2015). Segundo FANG et al. (2018), os riscos à saúde das florestas naturais estão cada vez mais intensos, favorecendo o declínio e a mortalidade destes ecossistemas em muitas regiões do planeta.

Em florestas temperadas e boreais, a temperatura ótima para a fotossíntese está entre $20{ }^{\circ} \mathrm{C}$ a $30 \stackrel{\circ}{\circ}$ (CUNNINGHAM \& READ 2003, VARGAS \& CORDERO 2013). Para TESKEY et al. (2015), temperaturas do ar acima de $40{ }^{\circ} \mathrm{C}$ passam a prejudicar os processos ecofisiológicos das plantas. O tempo de exposição é crucial, e se a temperatura for superior a $55^{\circ} \mathrm{C}$, haverá elevada taxa de respiração seguida de desidratação abrupta (ibid.).

STOVALL et al. (2019) observaram que as árvores de grande porte estão entre as mais suscetíveis. Segundo ALLEN (2009), a avaliação de declínio por meio de amarelecimento de copa e diebacks (ou ressecamento da biomassa foliar), é importante porque, apesar das árvores crescerem lentamente, podem morrer rapidamente e em grandes extensões de área.

\section{CONCLUSÃO}

O período noturno entre 21 e $3 \mathrm{~h}$ de 2020, da estação de verão de Dois Vizinhos, foi significativamente mais quente, no triênio analisado.

O horário entre 15 e 21 h de 2020 foi significativamente mais quente, entre as categorias horárias analisadas.

Por meio do índice FBS de estresse florestal, verificou-se o total de 36 episódios caracterizados como de Ondas de Calor na localidade, inclusive noturnas, entre os anos de 2018 a 2020.

\section{AGRADECIMENTOS}

O autor agradece ao INMET pela cessão de dados meteorológicos utilizados nesta pesquisa.

\section{REFERÊNCIAS}

ALLEN CD. 2009. Climate-induced forest dieback: an escalating global phenomenon? Unasylva 60: 231-232.

ALLEN CD et al. 2010. A global overview of drought and heat-induced tree mortality reveals emerging climate change risks for forests. Forest Ecology and Management 259: 660-684.

ALVARES CA et al. 2013. Köppen's climate classification map for Brazil. Meteorologische Zeitschrift 22: 711-728.

ALVES MPA et al. 2016. Caracterização da forte onda de calor de 2014 em Santa Catarina. Ciência e Natura 36: 309- 
325 .

AUBIN I et al. 2018. Tree vulnerability to climate change: improving exposure-based assessments using traits as indicators of sensitivity. Ecosphere 9: 1-24.

BITENCOURT DP et al. 2016. Frequência, duração, abrangência espacial e intensidade das ondas de calor no Brasil. Revista Brasileira de Meteorologia 31: 506-517.

BURAS A et al. 2020. Quantifying impacts of the 2018 drought on European ecosystems in comparison to 2003. Biogeosciences 17: 1655-1672.

CARDONA OD et al. 2012. Determinants of risk: exposure and vulnerability. In: FIELD CB et al. (Ed.). Managing the Risks of Extreme Events and Disasters to Advance Climate Change. Cambridge: Cambridge University Press. p.65108.

CASTRO FARIA AB et al. 2019. Pinheiros antigos podem entrar em declínio devido ao efeito das mudanças climáticas. BIOFIX 4: 16-25.

CUNNINGHAM SC \& READ J. 2003. Do temperate rainforest trees have a greater ability to acclimate to changing temperatures than tropical rainforest trees? The New Phytologist 157: 55-64.

FANG O et al. 2018. Tree rings reveal a major episode of Forest mortality in the late 18th century on the Tibetan Plateau. Global and Planetary Change 163: 44-50.

FIRPO MAF et al. 2012. Climatologia e variabilidade sazonal do número de ondas de calor e de frio no Rio Grande Do Sul associadas ao ENOS. Revista Brasileira de Meteorologia 27: 95-106.

GEIRINHAS JLM. 2016. Caracterização Climática e Sinóptica das Ondas de Calor no Brasil. Dissertação. (Mestrado em Geofísica). Lisboa: Universidade de Lisboa. 83p.

HARTMANN H et al. 2015. Research frontiers in drought-induced tree mortality: crossing scales and disciplines. New Phytologist 205: 965-969.

HARTMANN H et al. 2018. Monitoring global tree mortality patterns and trends. Report from the VW symposium "Crossing scales and disciplines to identify global trends of tree mortality as indicators of forest health". New Phytologist 217: 984-987.

IPCC. 2014. Intergovernmental Panel on Climate Change. Climate Change 2014: Synthesis Report - Contribution of Working Groups I, II and III to the Fifth Assessment Report of the IPCC. Genova: IPCC. 151p.

KIRKPATRICK SEP \& LEWIS SC. 2020. Increasing trends in regional heatwaves. Nature communications 11: 1-8.

KOGIMA KC \& ELY DF. 2019. Índices térmicos para a identificação de ondas de calor aplicados ao estado do Paraná, Brasil. Geo UERJ 34: 1-37.

MARENGO JA. 2014. O futuro clima do Brasil. Revista USP 103: 25-32.

MORALES MS et al. 2020. Six hundred years of South American tree rings reveal an increase in severe hydroclimatic eventos since mid- $20^{\text {th }}$ century. Proceedings of the National Academy of Sciences of the United States of America 117: 16816-16823.

RADINOVIÉ D \& CURIÉ M. 2012. Criteria for heat and cold wave duration indexes. Theoretical and Applied Climatology 107: 505-510.

SILVA WL et al. 2015. Tendências observadas em indicadores de extremos climáticos de temperatura e precipitação no estado do Paraná. Revista Brasileira de Meteorologia 30: 181-194.

STOVALL AEL et al. 2019. Tree height explains mortality risk during an intense drought. Nature Communications 10 : 4385.

TESKEY R et al. 2015. Responses of tree species to heat waves and extreme heat events. Plant, Cell and Environment 38: $1699-1712$.

TOMASETTO MZC et al. 2009. Desenvolvimento local e agricultura familiar: o caso da produção de açúcar mascavo em Capanema - Paraná. Interações 10: 21-30.

TRUMBORE S. et al. 2015. Forest health and global change. Science 349: 814-818.

VARGAS GG \& CORDERO SRA. 2013. Photosynthetic responses to temperature of two tropical rainforest tree species from Costa Rica. Trees 27: 1261-1270.

VIEIRA FMC et al. 2018. Probability distributions of frequency analysis of rainfall at the southwest region of Paraná State, Brazil. Revista de Ciências Agroveterinárias 17: 260-266.

VINCENT LA et al. 2005. Observed Trends in Indices of Daily Temperature Extremes in South America 1960-2000. Bulletin of the American Meteorological Society 18: 5011-5023.

WMO. 2015. World Meteorological Organization. Heatwaves and Health: Guidance on Warning-System Development. Genebra: WMO. 114p. 